\title{
Internações por doenças de veiculação hídrica: distribuição espacial e correlação no estado do Rio de Janeiro, 2008-2018
}

Objetivou-se identificar o padrão espacial de internações por doenças de veiculação hídrica no estado do Rio de Janeiro para o período de 2008 a 2018 , bem como analisar a correlação entre as taxas de internação pelo referido grupo de doenças e características sanitárias e socioeconômicas. A distribuição espacial das taxas de internação por doenças de veiculação hídrica permite que sejam delimitadas áreas prioritárias para a implementação de ações integradas, que contemplem a melhoria das condições de saneamento básico, principalmente no que se refere ao tratamento do esgoto coletado, além de ações que contribuam para a educação sanitária da população. Foram aplicadas, neste estudo, técnicas de análise espacial de dados de área (Índice de Moran) e análise de correlação de Spearman. Os resultados mostraram redução nas taxas de internação pelo grupo de doenças no estado. Entretanto, as regiões Noroeste e Serrana ainda apresentam municípios com elevadas taxas de internação e auto correlação espacial positiva, sendo identificadas como áreas prioritárias para adoção de medidas mitigadoras. As variáveis investimento realizado em coleta/tratamento de esgoto, atendimento por coleta com tratamento de esgoto, IDH, IDH Renda e taxa de alfabetização se correlacionaram negativamente com a taxa média de internação, enquanto o atendimento por coleta sem tratamento de esgoto apresentou correlação positiva. Cabe ressaltar, que a gestão deve sempre considerar as especificidades locais, para que as medidas sejam eficazes e a população seja protegida do acometimento dessas e outras enfermidades.

Palavras-chave: Poluição da água; Saneamento Básico; Saúde.

\section{Hospitalization for waterbone diseases: spatial distribution and correlation in the state of Rio de Janeiro, 2008-2018}

\begin{abstract}
The objective was to identify the spatial pattern of hospitalizations for waterborne diseases in the state of Rio de Janeiro for the period from 2008 to 2018 , as well as to analyze the correlation between hospitalization rates by the group of diseases and sanitary and socioeconomic characteristics. The spatial distribution of hospitalization rates for waterborne diseases allows priority areas to be defined for the implementation of integrated actions, which include the improvement of basic sanitation conditions, especially with regard to the treatment of collected sewage, in addition to actions that contribute to the health education of the population. Spatial analysis techniques of area data (Moran Index) and Spearman correlation analysis were applied in this study. The results showed a reduction in hospitalization rates by the group of diseases in the state. However, the Northwest and Serrana regions still have municipalities with high rates of hospitalization and positive spatial self-correlation, being identified as priority areas for the adoption of mitigating measures. The variables investment made in sewage collection/treatment, collection service with sewage treatment, HDI, HDI Income and literacy rate were negatively correlated with the average hospitalization rate, while collection service without sewage treatment showed a positive correlation. It should be noted that management must always consider local specificities, so that the measures are effective and the population is protected from being affected by these and other diseases.
\end{abstract}

Keywords: Water Pollution; Basic Sanitation; Health.

Topic: Epidemiologia e Saúde Ambiental

Reviewed anonymously in the process of blind peer.
Received: 04/07/2021

Approved: $27 / 07 / 2021$
Roberta Fernanda da Paz de Souza Paiva (iD

Universidade Federal Fluminense, Brasil

http://lattes.cnpq.br/3803580402918986

http://orcid.org/0000-0001-5856-822X

robertapaz2003@yahoo.com.br

Marcela Fernanda da Paz de Souza (i)

Universidade do Estado de Minas Gerais, Brasil

http://lattes.cnpq.br/6217112609060252

http://orcid.org/0000-0001-7437-5436

marcela.souza@uemg.br

Ana Luiza Maia (1D

Universidade Federal Fluminense, Brasil http://lattes.cnpq.br/0444660218204883

http://orcid.org/0000-0002-4186-5532

nalu.maia@hotmail.com
Carolina da Silva Rocha

Universidade Federal Fluminense, Brasil

http://lattes.cnpq.br/9286233664670581

caroolina.rocha@gmail.com
Referencing this:

PAIVA, R. F. P. S.; SOUZA, M. F. P.; MAIA, A. L.; ROCHA, C. S..

Internações por doenças de veiculação hídrica: distribuição espacial e correlação no estado do rio de janeiro, 2008-2018. Revista Ibero Americana de Ciências Ambientais, v.12, n.7, p.155-164, 2021. DOI: http://doi.org/10.6008/CBPC2179-6858.2021.007.0015 


\section{INTRODUÇÃO}

A manutenção da qualidade de vida da população depende do acesso aos recursos naturais com disponibilidade e qualidade adequados tendo, nesse processo, o saneamento ambiental papel fundamental (MOONEY et al., 2005). Nas regiões em que as condições de saneamento são inadequadas, tem-se mais elevado o risco de acometimento das pessoas por doenças relacionadas à poluição (FUNASA, 2010; KRONEMBERGER et al., 2011; FONTOURA et al., 2018).

Segundo os objetivos do desenvolvimento sustentável propostos pela Organização das Nações Unidas (ONU, 2015), a redução da poluição da água e o atendimento da equidade na quantidade e qualidade do recurso distribuído, além de melhorias nas condições de renda e higiene, contribuiriam para a melhoria das condições de saúde e aumento dos níveis de bem-estar da população, principalmente dos grupos mais vulneráveis. Esses benefícios se dariam não apenas com a redução dos casos de doenças de veiculação hídrica, mas ainda a partir de outras dimensões, como conforto, conveniência, privacidade, segurança, status social, entre outros (CAIRNCROSS, 2018).

Dados da Organização Mundial de Saúde (OMS) indicaram que melhores condições de saneamento básico e a adoção de práticas de higiene poderiam ter evitado cerca de $58 \%$ das mortes por diarreia no mundo em 2012. Segundo o relatório da Organização, bilhões de pessoas no mundo não possuem água adequada para consumo, serviços de esgotamento seguro e instalações básicas para a higienização das mãos (WHO et al., 2019).

Diversos estudos também concluíram sobre a associação entre condições sanitárias e socioeconômicas inadequadas e a morbidade e/ou mortalidade da população por doenças de veiculação hídrica no Brasil (GEERE et al., 2020; MUNAMATI et al., 2019) e no mundo (ALAGIDEDE et al., 2016; VAZ et al., 2017; PAIVA et al., 2018; PRÜSS-ÜSTÜN et al., 2019; FORGIARINI et al., 2020).

No Brasil, apesar dos investimentos realizados nos últimos anos, ainda não se tem a universalização do acesso aos serviços de saneamento básico. Dados do Censo Demográfico de 2010 indicaram que 82,85 \% dos domicílios do Brasil possuíam abastecimento de água por rede geral e, apenas, 55,5\% coleta de esgoto por rede geral (IBGE, 2010). Do total de esgoto coletado, segundo Sistema Nacional de Informações sobre Saneamento (BRASIL, 2016), apenas $44,9 \%$ é tratado antes do lançamento em corpos hídricos. As regiões Norte e Nordeste, que possuem os menores valores de pib per capita quando comparadas às demais regiões, são aquelas que possuem menor cobertura de domicílios com abastecimento de água e de coleta de esgoto por rede geral.

Nas duas últimas décadas, observou-se uma redução da ocorrência de internações por doenças de veiculação hídrica no Brasil. Entretanto, no período de 2008 a 2018, essas ainda representaram 3,2\% das internações do Sistema Único de Saúde, correspondendo a 0,9\% dos gastos totais com internações no período ( $\mathrm{R} \$ 1,9$ bilhões), constituindo um problema de saúde pública principalmente em determinadas localidades (BRASIL, 2020).

No estado do Rio de Janeiro, objeto do estudo, apesar de maior proporção de domićlios com 
abastecimento de água e coleta de esgoto por rede geral em relação ao Brasil $(84,58 \%$ e 76,59\% respectivamente), existem desigualdades entre seus 92 municípios, que vivenciam condições inadequadas de saneamento e seus impactos na qualidade de vida (IBGE, 2010). Esta disparidade se observa também nas taxas de internação por doenças de veiculação hídrica já que, enquanto no estado a taxa observada para o período de 2008 a 2018 foi de 1,2\%, seus municípios apresentaram taxas anuais que variaram entre 0,0\% e 95,01\%, sendo os gastos do estado com essas internações de $\mathrm{R} \$ 33,2$ milhões no período (BRASIL, 2020).

O entendimento da dinâmica espacial dos casos e dos fatores aos quais se relacionam permite que sejam realizadas intervenções locais e regionais, possibilitando que às áreas vulneráveis e de maior incidência sejam direcionados mais esforços no intuito de mitigar tais ocorrências no estado. As análises espaciais vêm sendo utilizadas como instrumento útil ao planejamento e gestão de ações de saúde para casos de tuberculose (BARBOSA et al., 2013), pneumonia (MENEZES et al., 2019), diarreia (MUNAMATI et al., 2019), doenças relacionadas ao saneamento ambiental inadequado (FONSECA et al., 2011), entre outros.

De forma complementar, destaca-se a importância do mapeamento de áreas de risco para doenças de veiculação hídrica e a proposição de solução a partir de cada realidade local como pontos essenciais ao atendimento das condições adequadas de quantidade e qualidade do recurso hídrico disponibilizado à população (GUERRA et al., 2018).

Diante do contexto apresentado, o objetivo desse estudo é identificar o padrão espacial de internações por doenças de veiculação hídrica no estado do Rio de Janeiro para os anos de 2008 e 2018, bem como analisar a correlação entre as taxas de internação pelo referido grupo de doenças e características sanitárias e socioeconômicas.

\section{METODOLOGIA}

Trata-se de um estudo do tipo ecológico e exploratório utilizando técnicas de análise espacial de dados de área e análise de correlação de Spearman. Foram consideradas internações por doenças de veiculação hídrica para o estado do Rio de Janeiro, sendo seus municípios as unidades de análise consideradas na pesquisa.

Segundo estado brasileiro em geração de renda (PIB), o Rio de Janeiro abriga 15.989.929 habitantes, tendo densidade demográfica de 365,23 hab/ $\mathrm{km}^{2}$ (IBGE, 2010). Apesar do PIB elevado e o IDH de 0,761 (IBGE, 2010), que o classifica como alto desenvolvimento humano, apresenta desigualdades quando consideradas suas características socioeconômicas e de saneamento, apresentando o maior Índice de Gini $(0,503)$ entre os estados da Região Sudeste e do Brasil (0,491) (IBGE, 2015).

As doenças consideradas foram selecionadas por estarem relacionadas à transmissão direta pela água, por meio da ingestão ou contato (ou penetração na pele) com ela, a saber (FUNASA, 2010): cólera, febres tifoides e paratifoide, shiguelose, amebíase, diarreia e gastroenterite de origem infecciosa presumível, outras doenças infecciosas intestinais, esquistossomose (Classificação Internacional de Doenças, 10a revisão - CID-10: A00-A09; B65). Os dados sobre as internações foram obtidos junto ao DATASUS ${ }^{17}$.

Para analisar a distribuição espacial das internações procedeu-se ao cálculo das taxas de internação 
anuais para o grupo de doenças (equação 1), considerando-se os anos de 2008 a 2018, segundo proposto por FUNASA (2010). Taxa de internação hospitalar por doenças de veiculação hídrica (por município) = número de internações por doenças de veiculação hídrica (por município) / população (por município) * 10.000 equação 1.

A partir do cálculo das taxas de internação foi avaliada sua distribuição espacial e identificados padrões de dependência espacial, por meio do cálculo do Índice de Moran Global, com seu respectivo pvalor. Esse índice, que varia de $-1 \mathrm{a}+1$, mede quanto o valor observado de um atributo em determinada região é dependente dos valores dessa mesma variável nas localizações vizinhas. Valores próximos de 1 indicam que existe semelhança entre os vizinhos próximos, enquanto valores negativos indicam que a semelhança não ocorre. Buscando uma análise mais detalhada dessa associação foi calculado o Índice de Moran Local, adotando-se a matriz de semelhança por contiguidade (MONTEIRO et al., 2004). Foram construídos mapas de distribuição das taxas de internação e mapa de Moran para os anos de 2008 e 2018 para o estado do Rio de Janeiro.

Foi calculado ainda o coeficiente de correlação de Spearman para a taxa média de internação por doenças de veiculação hídrica no período e variáveis sanitárias e socioeconômicas, a saber: cobertura de domicílios por abastecimento de água por rede geral; cobertura de domicílios com coleta de esgoto; Investimento em coleta e tratamento de esgoto (R\$) ; Índice de Atendimento com coleta e sem tratamento (\%); Índice de Atendimento com coleta e com tratamento (\%); IDH; IDH Renda; Percentual de Pobres, Taxa de Alfabetização. Esse coeficiente permite que sejam conhecidas a direção e a intensidade da correlação entre duas variáveis (FIELD, 2009).

Os dados de saneamento e taxa de alfabetização foram obtidos na base de dados do Censo Demográfico do IBGE, para o ano de 2010; as informações sobre o investimento em coleta e tratamento de esgoto e Índices de Atendimento foram obtidas junto ao SNIS, para o ano de 2013; os demais estão disponíveis no Atlas da Vulnerabilidade Social disponibilizado pelo IPEA, sendo calculados para o ano de 2010.

O software utilizado para as análises espaciais foi o Terraview 4.2.2. As análises descritivas e de correlação foram feitas com o auxílio do software SPSS 20. Foram consideradas significativas estatisticamente as relações que apresentaram nível de significância de 5\%, ou seja, p<0,05 (SIEGEL et al., 2006).

\section{RESULTADOS}

Entre os anos de 2008 e 2018 foram registradas nos 92 municípios do estado do Rio de Janeiro 87.947 internações por doenças de veiculação hídrica. A média por município foi de 956 casos, com desvio padrão de 1.869, sendo os valores mínimo e máximo de internações, de 9 e 11.975 ocorrências, respectivamente. A taxa média de internação no período foi de $11,16(\mathrm{DP}=13,10)$. Os valores mínimos de 0,54 e máximo de 62,05 dessas taxas indicam a disparidade entre os municípios em relação às ocorrências.

Considerando-se as internações nos anos de 2008 e 2018, observa-se uma redução nas taxas médias de internação, que vão de 16,4 (DP = 17,8) em 2008 a 5,47 (DP = 8,6) em 2018.

Os mapas mostram uma redução geral nas taxas de internação no estado quando comparados os 
anos de 2008 e 2018. As maiores taxas continuam concentradas na região Noroeste do estado, enquanto os municípios do Médio Paraíba e Centro-Sul apresentaram redução em relação ao ano de 2008.

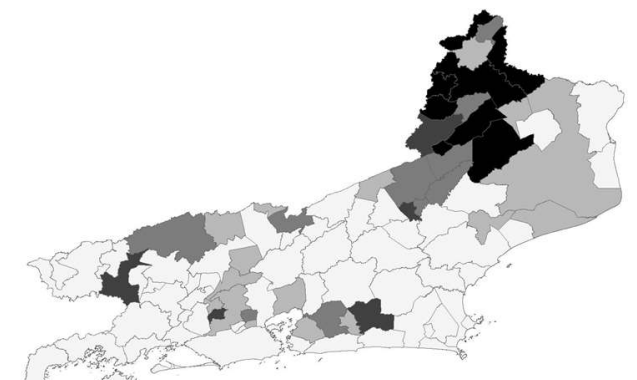

a)

Figura 1: Mapa temático da distribuição de taxas de internação por doenças de veiculação hídrica segundo os municípios do estado do Rio de Janeiro, 2008 (a) e 2018 (b).

Quanto aos fatores sanitários e socioeconômicos considerados no estudo observam-se diferentes condições entre os municípios do estado, principalmente no que se refere à cobertura por serviços de abastecimento de água e coleta de esgoto por rede geral.

Tabela 1: Análise descritiva das variáveis consideradas no estudo, com médias, desvio padrão, mínimo e máximo, para os municípios do estado do Rio de Janeiro.

\begin{tabular}{|c|c|c|c|c|}
\hline & Média & Desvio & Mínimo & Máximo \\
\hline \% Domicílios com Abastecimento de Água por rede geral & 71,34 & 19,56 & 18,95 & 98,87 \\
\hline \% Domicílios com Coleta de Esgoto por rede geral & 58,26 & 22,85 & 1,48 & 96,09 \\
\hline Investimento em coleta e tratamento de esgoto & $1,2157 \mathrm{E}+10$ & $3,47221 \mathrm{E}+10$ & $279.424 .310,00$ & $2,90 \mathrm{E}+11$ \\
\hline Índice de Atendimento com coleta e sem tratamento (\%) & 47,13 & 32,96 & 0 & 97 \\
\hline Índice de Atendimento com coleta e com tratamento & 21,65 & 31,79 & 0 & 100 \\
\hline IDHM & 0,7089 & 0,03677 & 0,61 & 0,84 \\
\hline IDHM Renda & 0,7046 & 0,04188 & 0,62 & 0,89 \\
\hline Taxa de Alfabetização (\%) & 93,24 & 3,32 & 83 & 99 \\
\hline
\end{tabular}

O Índice de Moran calculado para os anos de 2008 a 2018 indicou a presença de auto correlação espacial positiva em todos os anos, indicando que existe auto correlação espacial positiva das taxas de internação por doenças de veiculação hídrica no estado.

Tabela 2: Valores dos Índices de Moran, com p valor, para as taxas de internação por doenças de veiculação hídrica nos anos de 2008 a 2018.

\begin{tabular}{|c|c|c|}
\hline Taxas de Internação por doenças de veiculação hídrica (ano) & Índice Global de Moran & $p$-valor \\
\hline $\mathrm{TaxaDVH}_{2008}$ & 0,4795 & 0,01 \\
\hline $\mathrm{TaxaDVH}_{2009}$ & 0,4621 & 0,01 \\
\hline $\mathrm{TaxaDVH}_{2010}$ & 0,4489 & 0,01 \\
\hline $\mathrm{TaxaDVH}_{2011}$ & 0,4419 & 0,01 \\
\hline $\mathrm{TaxaDVH}_{2012}$ & 0,5285 & 0,01 \\
\hline $\mathrm{TaxaDVH}_{2013}$ & 0,4299 & 0,01 \\
\hline $\mathrm{TaxaDVH}_{2014}$ & 0,4403 & 0,01 \\
\hline $\mathrm{TaxaDVH}_{2015}$ & 0,3631 & 0,02 \\
\hline $\mathrm{TaxaDVH}_{2016}$ & 0,3694 & 0,01 \\
\hline $\mathrm{TaxaDVH}_{2017}$ & 0,3827 & 0,01 \\
\hline $\mathrm{TaxaDVH}_{2018}$ & 0,2740 & 0,01 \\
\hline
\end{tabular}

A partir do cálculo do índice foram construídos mapas de Moran para os anos de 2008 e 2018 . Os mapas indicam que houve mudanças na configuração dos municípios quanto a dependência espacial das internações.

No ano de 2008 a análise indicou que 20 municípios, localizados em sua maioria nas regiões Noroeste 
(65\%) e Serrana (20\%) do estado, estavam situados no quadrante 1 (Alto-Alto), sendo os que possuem altas taxas de internação e são cercados por vizinhos que também apresentam altas taxas de internação.

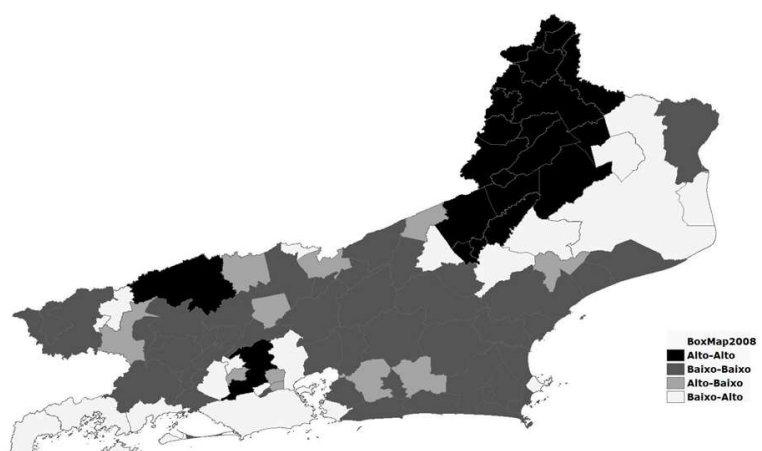

a)

Figura 2: Mapa de Moran para as taxas de internação de por doenças de veiculação hídrica no estado do Rio de Janeiro, por municípios, 2008 (a) e 2018 (b).

Os municípios de Nova Iguaçu, São Fidélis e Valença, das regiões Metropolitana, Norte e Médio Paraíba também apresentaram.

No ano de 2018, observaram-se mudanças na distribuição espacial das taxas de internação, com 17 municípios classificados no quadrante 1 , indicando uma redução em relação à 2008. As regiões nas quais se localizam a maioria desses municípios continuam sendo a Noroeste (47\%) e a Serrana (47\%), destacando-se o incremento de municípios na área da região Serrana e reduzindo a participação da Noroeste em relação a 2008. Apenas um município está localizado na região Norte (São Fidélis), fazendo divisa com municípios das regiões Nordeste e Serrana.

Os municípios de Aperibé, Bom Jesus do Itabapoana, Cambuci, Cantagalo, Cordeiro, Italva, Itaocara, Macuco, Miracema, Natividade, Santo Antônio de Pádua, São Fidélis e São Sebastião do Alto estavam classificados como Alto-Alto em 2008 e assim permaneceram na análise para 2018.

Deve-se destacar ainda os municípios que apresentam altas taxas de internação e são circundados por municípios com baixas taxas, como é o caso de Barra Mansa, Belford Roxo, Carmo, Conceição de Macabu, Itaboraí, Paty do Alferes, Queimados, Rio Bonito, Rio das Flores e Três Rios para 2008 e Barra Mansa, Belford Roxo, Quatis, Rio Bonito, Rio das Flores, Sumidouro e Tanguá para 2018.

Foi calculado o coeficiente de correlação de Spearmam, que indicou que as taxas médias de internação para os municípios estiveram correlacionadas negativamente ao investimento em coleta e tratamento de esgoto, Índice de Atendimento com coleta e com tratamento, IDH, IDH renda e à taxa de alfabetização, indicando que um aumento dessas variáveis estaria associado à uma redução das taxas de internação.

O Índice de Atendimento com coleta e sem tratamento apresentou correlação positiva, indicando que quanto maior o número de pessoas atendidas pela coleta de esgoto, mas sem o tratamento, maior tende a ser a taxa média de internação. 
Tabela 3: Coeficientes de correlação de Spearman para taxa média de internação e variáveis selecionadas.

\begin{tabular}{|l|l|l|}
\hline & Coeficiente de Correlação & P valor \\
\hline \%Domicílios com Abastecimento de Água por rede geral &,- 116 & 0,273 \\
\hline \%Domicílios com Coleta de Esgoto por rede geral &, 187 & 0,075 \\
\hline Investimento em coleta e tratamento de esgoto &,- 464 &, 000 \\
\hline Índice de Atendimento com coleta e sem tratamento (\%) &, 000 \\
\hline Índice de Atendimento com coleta e com tratamento &, 375 &, 004 \\
\hline IDHM &,- 295 &, 001 \\
\hline IDHM Renda &,- 351 &, 000 \\
\hline Taxa de Alfabetização &,- 467 &, 001 \\
\hline
\end{tabular}

\section{DISCUSSÃO}

A análise das internações por doenças de veiculação hídrica indicou que, apesar da redução observada no período de 2008 a 2018, alguns municípios do estado do Rio de Janeiro ainda apresentam elevadas taxas de internação pelo grupo de doenças. Esses municípios, em sua maioria, fazem parte das regiões Noroeste e Serrana do estado.

A região Noroeste abriga cerca de 1,9\% (334.097 habitantes) da população do estado (MENEZES et al., 2013) e concentrou, nos anos de 2008 e 2018, 13,8\% e 15,9\% das internações por doenças de veiculação hídrica do estado, respectivamente. Destaca-se, nessa região, o município de Bom Jesus do Itabapoana, cujas taxas de internação foram de 66,8 em 2008 e 61,1 em 2018, sendo a taxa média do período de 62,1. Observase que, no período analisado, houve apenas uma pequena redução na taxa de internação do município.

Na região Serrana, responsável por 5,3\% das internações no período, as taxas de internação permaneceram elevadas quando comparadas ao ano de 2018. Entretanto, alguns de seus municípios destacaram-se por apresentarem redução expressiva nas taxas de internação pelo referido grupo de doenças, como é o caso de Macuco (30,6 para 10,8) e Cordeiro (37,9 para 8,3).

Quando analisada a distribuição espacial das taxas de internação tem-se que municípios localizados nas regiões Noroeste e Serrana apresentaram dependência espacial para as internações. Dessa forma, encontram-se nessas áreas municípios que apresentam altas taxas de internação e são cercados por municípios que também possuem altas taxas. Observa-se ainda que 13 municípios que, em 2008, encontravam-se em áreas Alto-Alto, assim permaneceram em 2018. Isso indica que as regiões e não apenas os municípios precisam ser objetos de programas integrados que busquem melhorias nas condições de saúde no que tange o referido grupo de doenças.

A região Noroeste conta com $17,4 \%$ de sua população residente em área rural, maior percentual do estado e bem superior à média estadual, que é de 3,3\%. Estudo realizado indicou que a água de municípios dessa região não apresenta boa qualidade, sendo muitos dos corpos hídricos contaminados pela utilização de agrotóxicos nas atividades agrícolas (MENEZES et al., 2013).

Em estudo que buscou analisar o atendimento do Programa Vigilância de Qualidade da Água para o Consumo Humano (VIGIAGUA) no estado do Rio de Janeiro, Guerra et al. (2018) concluíram que essa região se destacou negativamente, possuindo a maioria dos municípios que realizavam parte ou nenhuma análise dos parâmetros de qualidade propostos pelo programa. Segundo os autores, o distanciamento geográfico dos municípios em relação ao Laboratório Central Noel Nutels (LACEN), localizado na cidade do Rio de 
Janeiro, e a fragilidade econômica da região são fatores que se refletem na atuação do VIGIAGUA na região. A Região Serrana também foi citada no estudo como uma das regiões que apresentaram maior quantitativo de municípios que não realizaram nenhuma das análises, ficando atrás apenas da região Noroeste.

Ainda sobre a qualidade da água, segundo o relatório do CEPERJ (BRASIL, 2019), apesar da maioria dos domicílios contarem com coleta de esgoto no estado, o sistema de saneamento básico permanece precário em grande parte de seus municípios. Os dados utilizados para o cálculo do ICMS ecológico, que considera as condições de coleta e de tratamento de esgoto, indicam que a cobertura da população por esgoto tratado na maioria dos municípios é muito baixa, sendo esse o caso das regiões Noroeste e Serrana.

A correlação negativa significativa entre os investimentos realizados pelos municípios em coleta e tratamento de esgoto e as taxas médias de internação encontrada neste estudo apresenta-se como um indicativo de que a elevação desses investimentos está relacionada à redução nas taxas de internação por doenças de veiculação hídrica. Outros autores já concluíram sobre a importância de condições adequadas de esgotamento sanitário (coleta e/ou tratamento), que são imprescindíveis à melhoria nas condições de saúde da população e redução da morbimortalidade por doenças de veiculação hídrica, seja para crianças (PRÜSSÜSTÜN et al., 2019; PAIVA et al., 2018; WOLF et al., 2018) ou para a população em geral (GEERE et al., 2020).

O mesmo pode ser concluído se avaliadas as correlações positiva e negativa encontradas, respectivamente, quando analisados os índices de atendimento com coleta e sem tratamento de esgoto e de atendimento com coleta e com tratamento do esgoto. Nesse caso, o atendimento da população pela coleta e tratamento do esgoto contribui para a redução das internações por doenças de veiculação hídrica, indicando a importância do tratamento do esgoto coletado.

Os resultados da correlação para a cobertura de domicílios por abastecimento de água por rede geral e cobertura de coleta de esgoto por rede geral não apresentaram significância estatística. Isso pode ter ocorrido porque, apesar da cobertura por esses serviços ter aumentado nos últimos anos, não se tem exato conhecimento da qualidade da água que efetivamente chega à população nesses municípios (MUNAMATI et al., 2019; BRASIL, 2019).

O IDH, IDH Renda e a taxa de alfabetização apresentaram correlação negativa com as taxas de internação, indicando que quanto maior for o desenvolvimento da região, os níveis de renda per capita e a o percentual de indivíduos alfabetizados melhores seriam as condições de vida da população, sendo observada a tendência de redução no acometimento da população pelas doenças de veiculação hídrica e, em consequência, nas internações.

O nível educacional da população e seu conhecimento acerca das condições de higiene e possíveis fontes de contaminação são fatores determinantes para minimizar o acometimento da mesma por doenças de veiculação hídrica. Assim a mitigação dessas doenças passa, além do investimento em melhorias das condições de moradia e sanitárias, da adoção de programas de educação sanitária (PIOLI et al., 2016; MIRANDA et al., 2016). 


\section{CONCLUSÕES}

A distribuição espacial das taxas de internação por doenças de veiculação hídrica permite que sejam delimitadas áreas prioritárias para a implementação de ações integradas, que contemplem a melhoria das condições de saneamento básico, principalmente no que se refere ao tratamento do esgoto coletado, além de ações que contribuam para a educação sanitária da população.

No estado do Rio de Janeiro, as regiões Noroeste e Serrana são prioritárias, devendo estado e municípios direcionarem esforços no sentido de melhorar os indicadores de saúde para as doenças relacionadas ao consumo e contato com a água. Os municípios para os quais as taxas de internação apresentaram redução devem manter a vigilância sobre os indicadores, buscando evitar a elevação do número de casos. Cabe ressaltar, que a gestão deve sempre considerar as especificidades locais, para que as medidas sejam eficazes e a população seja protegida do acometimento dessas e outras enfermidades.

AGRADECIMENTOS: À Fundação Carlos Chagas Filho de Amparo à Pesquisa do Estado do Rio de Janeiro (FAPERJ) pelo auxílio financeiro e bolsa de iniciação científica.

\section{REFERÊNCIAS}

ALAGIDEDE, P.; ALAGIDEDE, A. N.. The public health effects of water and sanitation in selected West African countries. Public Health, v.130, p.59-63, 2016. DOI: http://doi.org/10.1016/j.puhe.2015.07.037

BARBOSA, I. R.; PEREIRA L. M. S.; MEDEIROS, P. F. M.; VALENTIM, R. S.; BRITO, J. M.; COSTA, I. C. C.. Análise da distribuição espacial da tuberculose na região Nordeste do Brasil. Epidemiologia e Serviços de Saúde, Brasília, v.22, n.4, p.687-695, 2013. DOI: http://dx.doi.org/10.5123/S1679$\underline{49742013000400015}$

BRASIL. Diagnóstico dos serviços de Água e Esgoto - 201. Sistema Nacional de Informação sobre Saneamento. Brasília: SNIS, 2016.

BRASIL. Fundação Centro Estadual de Estatísticas, pesquisa e formação de servidores públicos do Rio de Janeiro. Aspectos da Qualidade de Vida do Estado do Rio de Janeiro: Olhar Comparativo. Rio de Janeiro: CEPERJ, 2019.

BRASIL. Morbidade Hospitalar do SUS: por local de residência: Brasil e municípios. Sistema de Informação Hospitalar: DATASUS, 2020.

CAIRNCROSS, S.. The public health benefits of urban sanitation in low and middle income countries. Utilities Policy, Londres, v.51, p.82-88, 2018. DOI: http://doi.org/10.1016/j.jup.2018.03.001

FIELD, A.. Descobrindo a estatística usando SPSS. Porto Alegre: Artmed, 2009.

FONSECA, F. R.; VASCONCELOS, C. H.. Análise Espacial das doenças relacionadas ao saneamento ambiental no Brasil. Cadernos Saúde Coletiva, Rio de Janeiro, v.19, n.4, p.448-53, 2011.
FONTOURA, V. M.; FONTOURA, I. G.; SANTOS, F. S.; SANTOS NETO, M.; TAVARES, H. S. A.; BEZERRA, M. O. L.; FEITOSA, M. O.; NEVES, A. F.; MORAIS, J. C. M.; NASCIMENTO, L. F. C.. Socio-environmental factors and diarrheal diseases in under five-year old children in the state of Tocantins, Brazil. Mark Simonds Riddle, Uniformed Services University of the Health Sciences, Washington, v.13, n.5, 2018. DOI: http://doi.org/10.1371/journal.pone.0196702

FORGIARINI, F. R.; PACHALY, R. L.; FAVARETTO, J.. Análises espaciais de doenças diarreicas e sua relação com o monitoramento ambiental. Associação Brasileira de Engenharia Sanitária e Ambiental, Santa Maria, v.23, n.5, p.963-972, 2018. DOI: http://dx.doi.org/10.1590/s1413$\underline{41522018169681}$

FUNASA. Fundação Nacional da Saúde. Impactos na saúde e no sistema único de saúde decorrentes de agravos relacionados a um saneamento ambiental inadequado. Brasília: FUNASA, 2010.

GEERE, J. A. L.; HUNTER, P. R.. The association of water carriage, water supply and sanitation usage with maternal and child health. A combined analysis of 49 multiple indicator cluster surveys from 41 countries. International Journal of Hygiene and Environmental Health, v.223, n.1, p.238-247, 2020. DOI: http://doi.org/10.1016/j.ijheh.2019.08.007

GUERRA, L. V.; SILVA, B. D.. Vigilância da Qualidade da água para Consumo no Estado do Rio de Janeiro. Ambiente \& Sociedade, São Paulo, v.21, 2018. DOI: http://dx.doi.org/10.1590/1809-4422asoc0097r2vu18|3td

IBGE. Instituto Brasileiro de Geografia e Estatística. Censo Brasileiro de 2010. Brasília: IBGE, 2010.

IBGE. Instituto Brasileiro de Geografia e Estatística. 
Indicadores de Desenvolvimento Sustentável. Brasília: IBGE, 2015.

KRONEMBERGER, D. M. P.; PEREIRA, R. S.; FREITAS, E. A. V.; SCARCELLO, J. A.; CLEVELARIO JUNIOR, J.. Saneamento e meio ambiente. Rio de Janeiro: Atlas de Saneamento - IBGE, 2011.

MENEZES, J. M.; SILVA JUNIOR, G. C.; PRADO, R. B.. Índice de qualidade de água (IQACCME) aplicado à avaliação de aquíferos do estado do Rio de Janeiro. Águas Subterrâneas, Rio de Janeiro, v.27, n.2, 2013.

MENEZES, R. A. M.; PAVANITTO, D. R.; NASCIMENTO, L. F. C. Distribuição espacial das taxas de internação de crianças por pneumonia no Sistema Único de Saúde, nos municípios do estado de São Paulo. Revista Brasileira de Epidemiologia, São Paulo, v.22, p.1-10, 2019. Dol: http://doi.org/10.1590/1980-549720190053

MIRANDA, J. P. R.; GARCÍA-UBAQUE, C. A.; GARCÍA-UBAQUE, J. C.. Enfermedades transmitidas por el agua y saneamiento básico en Colombia. Revista de Salud Pública, v.18, p.738745, 2016. DOI: http://doi.org/10.15446/rsap.v18n5.54869

MONTEIRO, A. M. V.; CÂMARA, G.; CARVALHO M. S.; DRUCK S.. Análise espacial de dados geográficos. Brasília: Embrapa, 2004.

MOONEY, H. A.; CROPPER, A.; CAPISTRANO, D.; CARPENTER, S. R.; CHOPRA, K.; DASGUPTA, P.; HASSAN, R.; LEEMANS, R.; MAY, R. M.; PINGALI, P.; SAMPER, C.; SCHOLES, R.; WATSON, R. T.; ZAKRI, A. H.; SHIDONG, Z.. Ecosystems and human well-being: Health Synthesis. Millennium Ecosystem Assessment Board, 2005.

MUNAMATI, M.; NHAPI, I.; MISI, S.. Exploring the sanitation success, sanitation technology and diarrhoeal mortality nexus in Sub-Saharan Africa. Physics and Chemistry of the Earth, Parts A/B/C, v.14, 2019. DOI: http://doi.org/10.1016/j.pce.2019.08.003

ONU. Organização das Nações Unidas. Objetivos de Desenvolvimento Sustentável: Brasília: ONU, 2015.
PAIVA, R. F. P. S.; SOUZA, M. F. P.. Associação entre condições socioeconômicas, sanitárias e de atenção básica e a morbidade hospitalar por doenças de veiculação hídrica no Brasil. Cadernos de Saúde Pública, Volta Redonda, v.34 2018. DOI: http://dx.doi.org/10.1590/0102-311x00017316

PIOLI, M.; PIRES, R. H.; RAMO, S. B.; MARTINS, C. H.; APARECIDO, L. E. O.; ZAIA, J. E.. Influência de fatores de risco na mortalidade por doenças infecciosas e parasitárias. Saúde e Pesquisa, Maringá, v.9, n.3, p.491-498, 2016. DOI: http://dx.doi.org/10.177651/1983-1870.2016v9n3p491-498

PRÜSS-USTÜN, A.; WOLF, J.; BARTRAM, J.; CLASEN, T.; CUMMING, O.; FREMMAN, M. C.; GORDON, B.; HUNTER, P. R.; MEDLICOTT, K.; JOHNSTON, R.. Burden of disease from inadequate water, sanitation and hygiene for selected adverse health outcomes: an updated analysis with a focus on low-and middle-income countries. International Journal of Hygiene and Environmental Health, v.222, n.5, p.765777, 2019. DOI: http://doi.org/10.1016/j.ijheh.2019.05.004

SIEGEL, S.; CASTELLAN JUNIOR, N. J.. Estatística nãoparamétrica para ciências do comportamento. 2 ed. São Paulo: Artmed, 2006.

VAZ, F. P. C.; NASCIMENTO, L. F. C.. Spatial distribution for diarrhea hospitalization in São Paulo State. Revista Brasileira de Saúde Materno Infantil, Recife, v.17, n.3, p.475-482, 2017. DOI: http://doi.org/10.1590/1806$\underline{93042017000300004}$

WHO; UNICEF. World Health Organization; United Nations Children's Fund. Progress on household drinking water, sanitation and hygiene 2000-2017. Special focus on inequalities. New York: WHO, 2019.

WOLF, J.; HUNTER, P. R.; FREEMAN, M. C.; CUMMING, O.; CLASEN, T.; BARTRAM, J.; HIGGINS, J. P. T.; JOHNSTON, R.; MEDLICOTT, K.; BOISSON, S.; PRÜSS-USTÜN, A.. Impact of drinking water, sanitation and hand washing with soap on childhood diarrhoeal disease: updated meta-analysis andregression. Tropical Medicine and International Health, v.23, n.5, p.508-525, 2018. DOI: http://doi.org/10.1111/tmi.13051

A CBPC - Companhia Brasileira de Produção Científica (CNPJ: 11.221.422/0001-03) detém os direitos materiais desta publicação. Os direitos referem-se à publicação do trabalho em qualquer parte do mundo, incluindo os direitos às renovações, expansões e disseminações da contribuição, bem como outros direitos subsidiários. Todos os trabalhos publicados eletronicamente poderão posteriormente ser publicados em coletâneas impressas sob coordenação da Sustenere Publishing, da Companhia Brasileira de Produção Científica e seus parceiros autorizados. Os (as) autores (as) preservam os direitos autorais, mas não têm permissão para a publicação da contribuição em outro meio, impresso ou digital, em português ou em tradução. 